\title{
A NOTE ON NORM INEQUALITIES FOR INTEGRAL OPERATORS ON CONES
}

\author{
KECHENG ZHOU
}

\begin{abstract}
Norm inequalities for the Riemann-Liouville operator $R_{r} f(x)=$ $\int_{\langle 0, x\rangle} \Delta_{V}^{r-1}(x-t) f(t) d t$ and Weyl operator $W_{r} f(x)=\int_{\langle x, \infty\rangle} \Delta_{V}^{r-1}(t-x) f(t) d t$ on cones in $R^{d}$ have been obtained in the case $r \geq 1$ [7]. In this note, these inequalities are further extended to the case $r<1$. The question of whether the Hardy operator $H f(x)=\int_{\langle 0, x\rangle} f(t) d t$ on cones is bounded from $L^{p}\left(\Delta_{V}^{\alpha}(x)\right)$ to $L^{q}\left(\Delta_{V}^{\beta}(x)\right) \quad(q<p)$ is also solved.
\end{abstract}

Let $V$ be a homogeneous cone in $R^{d}$. $V$ defines a partial ordering in $R^{d}$ in such a way that $x<_{V} y$ if and only if $y-x \in V$. The cone interval $\langle a, b\rangle$ is thus given by $\langle a, b\rangle=\left\{x \in V: a<_{V} x<_{V} b\right\}$. For $x \in V$ we define $\Delta_{V}(x)=\int_{\langle 0, x\rangle} d y$.

Let $G(V)$ denote the automorphism group of $V$, and let $\Sigma=\{x \in V:|x|=$ 1\}, $\sigma_{0}=\sigma_{0}(V)=\inf \left\{\alpha: \int_{\Sigma} \Delta^{\alpha}\left(t^{\prime}\right) d t^{\prime}<\infty\right\}$ and $\sigma(V)=\max \left(-1, \sigma_{0}\right)$. It is known (see $[4,7])$ that if $\alpha>\sigma(V)$, then $\int_{\langle 0, x\rangle} \Delta_{V}^{\alpha}(t) d t$ is finite for all $x \in V$ and homogeneous of order $\alpha+1$ so that

$$
\int_{\langle 0, x\rangle} \Delta_{V}^{\alpha}(t) d t=c \Delta_{V}^{\alpha+1}(x) .
$$

The dual $V^{*}$ of $V$ is defined as $V^{*}=\left\{x \in R^{d}: x \cdot y>0, \forall y \in \bar{V}, y \neq 0\right\}$. Clearly, $V^{*}$ is also a cone. It is known that $V^{* *}=V$.

The $*$-function on $V$ is the mapping $x \rightarrow x^{*}$ such that $x^{*}=-\operatorname{grad} \log \phi(x)$, where $\phi(x)=\int_{V^{*}} e^{x \cdot y} d y$ is the characteristic function of $V$. It is known (see $[2,6])$ that the $*$-function is a one-to-one mapping from $V$ onto $V^{*}$. Let $G\left(V \rightarrow V^{*}\right)$ be the group of linear transformations mapping $V$ onto $V^{*}$. A homogeneous cone $V$ is said to be a domain of positivity if there is an element $S \in G\left(V \rightarrow V^{*}\right)$ so that $S$ is symmetric and positive definite. It can be shown (see $[6,7])$ that for a domain of positivity $V, x<_{V} y$ if and only if $y^{*}<_{V} \cdot x^{*}$.

In this note, we shall continue to consider the Riemann-Liouville operator

$$
R_{r} f(x)=\int_{\langle 0, x\rangle} \Delta_{V}^{r-1}(x-t) f(t) d t
$$

and Weyl operator

$$
W_{r} f(x)=\int_{\langle x, \infty\rangle} \Delta_{V}^{r-1}(t-x) f(t) d t
$$

Received by the editors April 26, 1993 and, in revised form, December 2, 1993; originally communicated to the Proceedings of the AMS by Palle E. T. Jorgensen.

1991 Mathematics Subject Classification. Primary 44A15. 
on cones on $R^{d}$. It is worth noting that the Riemann-Liouville operators whose kernels are complex power functions associated with the cone $V$ were extensively studied in [1], although they are different from the Riemann-Liouville operator we shall study here.

Theorem 1. Let $V$ be a domain of positivity in $R^{d}$. If $1 \leq p \leq q<\infty$, $r-1>\sigma(V)$, and $\gamma<-\sigma(V)\left(1+\frac{q}{p^{\prime}}\right)-\sigma\left(V^{*}\right)+q\left(\frac{1}{p}-r+1\right)-3$, then for any $f: V \rightarrow R^{+}$,

$$
\left(\int_{V} \Delta_{V}^{\gamma-q}(x)\left(R_{r} f(x)\right)^{q} d x\right)^{1 / q} \leq c\left(\int_{V} f^{p}(x) \Delta_{V}^{(r-1) p+(\gamma+1) p / q-1}(x) d x\right)^{1 / p}
$$

We show (1) in the case $r \geq 1$ (see [7]). Since $-1 \leq \sigma(V) \leq 0$ for any cone $V$, Theorem 1 extends the result to the case $0<r<1$. It is also worth noting that $\Delta_{V}(x) \rightarrow 0$ as $x$ approaches the boundary of $V$. Hence, the kernel of $R_{r}$ approaches infinity as $t$ approaches any pont on the boundary of $x-V$ in the case $r-1>\sigma(V)$. In the one dimensional case, where $V=(0, \infty)$, $\sigma(V)=-1, r>0$, Theorem 1 gives the boundedness of the Riemann-Liouville operator on the half line. form

In the proof of Theorem 1 we shall deal with integrals on the cone of the

$$
\int_{\langle 0, x\rangle} \Delta_{V}^{\alpha}(x-t) \Delta_{V}^{\beta}(t) d t
$$

and

$$
\int_{\langle x, \infty\rangle} \Delta_{V}^{\alpha}(t-x) \Delta_{V}^{\beta}(t) d t
$$

where $\alpha, \beta<0$. The following two lemmas prove that, under certain conditions on $\alpha$ and $\beta$, the integrals are finite for each $x \in V$ so that they can be "integrated" out. When restricted to the one dimensional case, these two lemmas give the best results.

Lemma 1. Let $V$ be a homogeneous cone and let

$$
g(x)=\int_{\langle 0, x\rangle} \Delta_{V}^{\alpha}(x-t) \Delta_{V}^{\beta}(t) d t, \quad x \in V .
$$

If $\alpha>\sigma(V)$ and $\beta>\sigma(V)$, then $g(x)$ is finite for each $x \in V$ and is homogeneous of order $\alpha+\beta+1$. Hence, there is a constant $c$ for which

$$
g(x)=c \Delta_{V}^{\alpha+\beta+1}(x), \quad x \in V .
$$

Proof. Let $y \in V$. By Fubini's theorem, we have

$$
\begin{aligned}
\int_{\langle 0, y\rangle} g(x) d x & =\int_{\langle 0, y\rangle} \Delta_{V}^{\beta}(t)\left(\int_{\langle t, y\rangle} \Delta_{V}^{\alpha}(x-t) d x\right) d t \\
& =\int_{\langle 0, y\rangle} \Delta_{V}^{\beta}(t)\left(\int_{\langle 0, y-t\rangle} \Delta_{V}^{\alpha}(z) d z\right) d t \\
& \leq \int_{\langle 0, y\rangle} \Delta_{V}^{\beta}(t)\left(\int_{\langle 0, y\rangle} \Delta_{V}^{\alpha}(z) d z\right) d t \\
& =\left(\int_{\langle 0, y\rangle} \Delta_{V}^{\beta}(t) d t\right) \cdot\left(\int_{\langle 0, y\rangle} \Delta_{V}^{\alpha}(z) d z\right) .
\end{aligned}
$$


If $\alpha>\sigma(V)$ and $\beta>\sigma(V)$, the two integrals above are finite, and so $g(x)$ is finite for almost every $x \in V$.

Let $x_{0} \in V$ be such that $g\left(x_{0}\right)$ is finite. Since $V$ is homogeneous, for any $x \in V$ there exists $A \in G(V)$ so that $x=A x_{0}$. Then we have

$$
\begin{aligned}
g(x) & =g\left(A x_{0}\right)=\int_{\left\langle 0, A x_{0}\right\rangle} \Delta_{V}^{\alpha}\left(A x_{0}-t\right) \Delta_{V}^{\beta}(t) d t \\
& =\int_{\left\langle 0, x_{0}\right\rangle} \Delta_{V}^{\alpha}\left(A\left(x_{0}-z\right)\right) \Delta_{V}^{\beta}(A z)|A| d z \\
& =\int_{\left\langle 0, x_{0}\right\rangle}|A|^{\alpha} \Delta_{V}^{\alpha}\left(x_{0}-z\right)|A|^{\beta} \Delta_{V}^{\beta}(z)|A| d z=|A|^{\alpha+\beta+1} g\left(x_{0}\right) .
\end{aligned}
$$

Hence, $g(x)$ is finite for each $x \in V$ and is homogeneous of order $\alpha+\beta+1$. Therefore, there is a constant $c$ for which $g(x)=c \Delta_{V}^{\alpha+\beta+1}(x), x \in V$.

Lemma 2. Let $V$ be a domain of positivity and let

$$
h(x)=\int_{\langle x, \infty\rangle} \Delta_{V}^{\alpha}(t-x) \Delta_{V}^{\beta}(t) d t, \quad x \in V .
$$

If $\alpha>\sigma(V)$ and $\alpha+\beta<-3-\sigma\left(V^{*}\right)-\sigma(V)$, then $h(x)$ is finite for each $x \in V$ and homogeneous of order $\alpha+\beta+1$. hence, there is a constant $c$ for which

$$
h(x)=c \Delta_{V}^{\alpha+\beta+1}(x), \quad x \in V .
$$

Proof. The condition on $\alpha+\beta$ gives $-\sigma(V)>3+\sigma\left(V^{*}\right)+\alpha+\beta$. Let $\gamma \in R$ so that $-\sigma(V)>\gamma>3+\sigma\left(V^{*}\right)+\alpha+\beta$. We have, for $y \in V$,

$$
\begin{aligned}
\int_{\langle y, \infty\rangle} & \Delta_{V}^{-\gamma}(x) h(x) d x \\
= & \int_{\langle y, \infty\rangle} \Delta_{V}^{-\gamma}(x) \int_{\langle x, \infty\rangle} \Delta_{V}^{\alpha}(t-x) \Delta_{V}^{\beta}(t) d t d x \\
= & \int_{\langle y, \infty\rangle}\left(\int_{\langle y, t\rangle} \Delta_{V}^{\alpha}(t-x) \Delta_{V}^{-\gamma}(x) d x\right) \Delta_{V}^{\beta}(t) d t \\
& \leq \int_{\langle y, \infty\rangle}\left(\int_{\langle 0, t\rangle} \Delta_{V}^{\alpha}(t-x) \Delta_{V}^{-\gamma}(x) d x\right) \Delta_{V}^{\beta}(t) d t .
\end{aligned}
$$

Since $\alpha>\sigma(V)$ and $-\gamma>\sigma(V)$, by Lemma 1, we have

$$
\int_{\langle y, \infty\rangle}\left(\int_{\langle 0, t\rangle} \Delta_{V}^{\alpha}(t-x) \Delta_{V}^{-\gamma}(x) d x\right) \Delta_{V}^{\beta}(t) d t=c \int_{\langle y, \infty\rangle} \Delta_{V}^{\alpha+\beta-\gamma+1}(t) d t .
$$

Since $V$ is a domain of positivity, a change of variable $t \rightarrow t^{*}$ gives

$$
\int_{\langle y, \infty)} \Delta_{V}^{\alpha+\beta-\gamma+1}(t) d t=\int_{\left\langle 0, y^{*}\right\rangle} \Delta_{V^{*}}^{-\alpha-\beta+\gamma-3}(t) d t .
$$

Since $-\alpha-\beta+\gamma-3>\sigma\left(V^{*}\right)$, the last integral is finite. Hence, $h(x)$ is finite for almost every $x \in V$. Clearly, $h(A x)=|A|^{\alpha+\beta+1} h(x)$ for $A \in G(V)$. Hence, $h(x)$ is finite for each $x \in V$ and is homogeneous of order $\alpha+\beta+1$. Therefore, there is a constant $c$ for which $h(x)=c \Delta_{V}^{\alpha+\beta+1}(x), x \in V$. 
Proof of Theorem 1. Noting the condition on $\gamma$ in the hypothesis, we can choose $b$ so that $\sigma(V)<b<\left(-3-\sigma\left(V^{*}\right)-\sigma(V)-\gamma+q\left(\frac{1}{p}-r+1\right)\right) \frac{p^{\prime}}{q}$.

Using Hölder's inequality, we have

$$
\begin{aligned}
& \int_{V} \Delta^{\gamma-q}(x)\left(R_{r} f(x)\right)^{q} d x \\
& =\int_{V} \Delta^{\gamma-q}(x)\left(\int_{\langle 0, x\rangle} \Delta_{V}^{(r-1) / p}(x-t) f(t)\right. \\
& \left.\cdot \Delta_{V}^{-b / p^{\prime}}(t) \Delta_{V}^{(r-1) / p^{\prime}}(x-t) \Delta_{V}^{b / p^{\prime}}(t) d t\right)^{q} d x \\
& \leq \int_{V} \Delta^{\gamma-q}(x)\left(\int_{\langle 0, x\rangle} \Delta_{V}^{r-1}(x-t) f^{p}(t) \Delta_{V}^{-b(p-1)}(t) d t\right)^{q / p} \\
& \cdot\left(\int_{\langle 0, x\rangle} \Delta_{V}^{r-1}(x-t) \Delta_{V}^{b}(t) d t\right)^{q / p^{\prime}} d x .
\end{aligned}
$$

Noting that $r-1>\sigma(V)$ and $b>\sigma(V)$, by Lemma 1 , we have

$$
\begin{aligned}
& \int_{V} \Delta^{\gamma-q}(x)\left(R_{r} f(x)\right)^{q} d x \\
& \quad \leq c \int_{V} \Delta_{V}^{\gamma-q+(r+b) q / p^{\prime}}(x)\left(\int_{\langle 0, x\rangle} \Delta_{V}^{r-1}(x-t) f^{p}(t) \Delta_{V}^{-b(p-1)}(t) d t\right)^{q / p} d x .
\end{aligned}
$$

Since $q / p \geq 1$, by the Minkowski integral inequality, we have

$$
\begin{aligned}
\int_{V} \Delta^{\gamma-q}(x)\left(R_{r} f(x)\right)^{q} d x \\
\leq c\left(\int_{V} f^{p}(t)\right. \\
\left.\quad \cdot \Delta_{V}^{-b(p-1)}(t)\left(\int_{\langle t, \infty\rangle} \Delta_{V}^{(r-1) q / p}(x-t) \Delta_{V}^{\gamma-q+(r+b) q / p^{\prime}}(x) d x\right)^{p / q} d t\right)^{q / p} .
\end{aligned}
$$

Noting that $(r-1) q / p>\sigma(V)$ and $(r-1) q / p+\gamma-q+(r+b) q / p^{\prime}=$ $r q-q / p+\gamma-q+b q / p^{\prime}<-3-\sigma\left(V^{*}\right)-\sigma(V)$, by Lemma 2 , we have

$$
\begin{aligned}
\int_{V} \Delta^{\gamma-q}(x)\left(R_{r} f(x)\right)^{q} d x \\
=c\left(\int_{V} f^{p}(t) \Delta_{V}^{-b(p-1)}(t) \Delta_{V}^{\left((r-1) q / p+\gamma-q+(r+b) q / p^{\prime}+1\right) p / q}(t) d t\right)^{q / p} \\
=c\left(\int_{V} f^{p}(t) \Delta_{V}^{(r-1) p+(\gamma+1) p / q-1}(t) d t\right)^{q / p} .
\end{aligned}
$$

Using Theorem 1 and the fact that Weyl's operator is the dual of RiemannLiouville's operator, we can prove the following norm inequality for Weyl's operator on cones. 
Theorem 2. Let $V$ be a domain of positivity in $R^{n}$. If $1 \leq p \leq q<\infty$, $r-1>\sigma(V)$, and $\gamma>\sigma(V)\left(1+q / p^{\prime}\right)+\sigma\left(V^{*}\right) q / p^{\prime}+q\left(1+2 / p^{\prime}\right)$, then

$$
\left(\int_{V} \Delta_{V}^{y-q}(x)\left(W_{r} f(x)\right)^{q} d x\right)^{1 / q} \leq c\left(\int_{V} f^{p}(x) \Delta_{V}^{(r-1) p+(\gamma+1) p / q-1}(x) d x\right)^{1 / p}
$$

Now we consider the Hardy operator

$$
H f(x)=\int_{\langle 0, x\rangle} f(t) d t
$$

on cones in $R^{d}$. As a corollary of the main theorem in [7], we have shown that if $1 \leq p \leq q \leq \infty$ and $\gamma<-\sigma(V) q / p^{\prime}-\sigma\left(V^{*}\right)+q / p-2$, then

$$
\left(\int_{V} \Delta_{V}^{\gamma-q}(x)(H f(x))^{q} d x\right)^{1 / q} \leq c\left(\int_{V} f^{p}(x) \Delta_{V}^{(\gamma+1) p / q-1}(x) d x\right)^{1 / p} .
$$

It is natural to inquire whether there exist appropriate numbers $\alpha$ and $\beta$ so that

$$
\left(\int_{V} \Delta^{\beta}(x)(H f(x))^{q} d x\right)^{1 / q} \leq c\left(\int_{V} f^{p}(x) \Delta^{\alpha}(x) d x\right)^{1 / p}
$$

holds for all $f \geq 0$ when $1 \leq q<p<\infty$. In the one dimensional case, the fact that (3) does not hold for any values of $\alpha$ and $\beta$ when $1 \leq q<p<\infty$ is simply a consequence of a theorem in [3] concerning the Hardy inequality with general weights. This result can be generalized to cones in $R^{d}$.

Theorem 3. Let $V$ be a domain of positivity in $R^{d}$. If $1 \leq q<p<\infty$, then for any values $\alpha$ and $\beta$, there is no constant $c>0$ such that (3) holds for all $f \geq 0$.

Using the Hardy operator with weight, we see immediately that Theorem 3 is equivalent to the following theorem.

Theorem 4. Let

$$
H_{\alpha} f(x)=\int_{\langle 0, x\rangle} f(t) \Delta_{V}^{\alpha}(t) d t
$$

and let $V$ be a domain of positivity in $R^{d}$. If $1 \leq q<p<\infty$, then for any values of $\alpha$ and $\beta$, there is no constant $c>0$ such that

$$
\left(\int_{V} \Delta_{V}^{\beta}(x)\left(H_{\alpha} f(x)\right)^{q} d x\right)^{1 / q} \leq c\left(\int_{V} f^{p}(x) \Delta_{V}^{\alpha}(x) d x\right)^{1 / p}
$$

holds for all $f \geq 0$.

Proof of Theorem 4. First we show that in order that a $c>0$ exist for which (4) holds for all $f \geq 0, \alpha$ and $\beta$ must satisfy $(\alpha+1) / p^{\prime}+(\beta+1) / q=0$ and $\beta<-1$.

Assume that (4) holds for some values of $\alpha$ and $\beta$. Then (4) implies that for all $z \in V$,

$$
\left(\int_{\langle z, \infty\rangle} \Delta_{V}^{\beta}(x)\left(\int_{\langle 0, x\rangle} f(t) \Delta_{V}^{\alpha}(t) d t\right)^{q} d x\right)^{1 / q} \leq c\left(\int_{V} f^{p}(x) \Delta_{V}^{\alpha}(x) d x\right)^{1 / p} .
$$


Further, it implies

$$
\left(\int_{\langle 0, z\rangle} f(t) \Delta_{V}^{\alpha}(t) d t\right) \cdot\left(\int_{\langle z, \infty\rangle} \Delta_{V}^{\beta}(x) d x\right)^{1 / q} \leq c\left(\int_{V} f^{p}(x) \Delta_{V}^{\alpha}(x) d x\right)^{1 / p} .
$$

Choose a sequence $\left\{V_{n}\right\}$ of nested cone intervals so that $\bar{V}_{n} \subset\langle 0, z\rangle$ and $V_{n} \nearrow\langle 0, z\rangle$. Note that $\int_{V_{n}} \Delta_{V}^{\alpha}(t) d t<\infty$. Let $f_{n}(x)=\chi_{V_{n}}(x)$. Substituting $f_{n}(x)$ in (5) we have

$$
\left(\int_{V_{n}} \Delta_{V}^{\alpha}(t) d t\right)^{1 / p^{\prime}} \cdot\left(\int_{\langle z, \infty\rangle} \Delta_{V}^{\beta}(x) d x\right)^{1 / q} \leq c .
$$

It follows that $\int_{V_{n}} \Delta_{V}^{\alpha}(t) d t$ is bounded and so $\int_{\langle 0, z\rangle} \Delta_{V}^{\alpha}(x) d x$ is finite. It also follows from (6) that $\int_{\langle z, \infty\rangle} \Delta_{V}^{\beta}(x) d x$ is finite for each $z$. It is known [6] that

$$
\Delta_{V}(x) \leq \rho|x|^{d} \text { for some } \rho>0 .
$$

Therefore, in order that $\int_{\langle z, \infty\rangle} \Delta_{V}^{\beta}(x) d x$ be finite for each $z$ it is necessary that $\beta<-1$.

Let $f(x)=\chi_{\langle 0, z\rangle}(x)$. Substituting $f(x)$ in (5) we have

$$
\left(\int_{\langle 0, z\rangle} \Delta_{V}^{\alpha}(t) d t\right)^{1 / p^{\prime}} \cdot\left(\int_{\langle z, \infty\rangle} \Delta_{V}^{\beta}(x) d x\right)^{1 / q} \leq c, \quad z \in V .
$$

Integrating these integrals in (8) and taking the supremum over $z \in V$, we have

$$
\sup _{z \in V} \Delta_{V}^{(\alpha+1) / p^{\prime}}(z) \Delta_{V}^{(\beta+1) / q}(z) \leq c .
$$

Therefore, it is necessary that $(\alpha+1) / p^{\prime}+(\beta+1) / q=0$.

Next, we show that (4) cannot hold even if $\alpha$ and $\beta$ satisfy the aforementioned conditions. First assume that $1<q$. Let $a=(q-1) /(p-q)$ and $b=q /(p-q)$. Note that $a>0, b>0$, and $(\alpha+1)(a+1 / p)+(\beta+1) b=0$. Take $z_{0} \in V$ with $\Delta_{V}\left(z_{0}\right)=1$. Define

$$
f_{n}(x)=\Delta_{V}^{a(\alpha+1)}(x) \min \left(n, \Delta_{V}^{b(\beta+1)}(x)\right) \chi_{\left\langle 0, n z_{0}\right\rangle}(x), \quad x \in V, n=1,2, \ldots
$$

Clearly, for each $n, f_{n}^{p}(x)$ is integrable on $V$. We show that $\int_{V} f_{n}^{p}(x) d x \rightarrow \infty$ as $n \rightarrow \infty$.

Choose $\alpha_{n}$ so that

$$
\Delta_{V}^{b(\beta+1)}\left(\alpha_{n} z_{0}\right)=\alpha_{n}^{d b(\beta+1)}=n .
$$

Then we have

$$
\begin{aligned}
\int_{V} f_{n}^{p}(x) \Delta_{V}^{\alpha}(x) d x \\
\quad \geq \int_{\left\langle\alpha_{n} z_{0}, n z_{0}\right\rangle} \Delta_{V}^{a(\alpha+1) p}(x) \cdot \Delta_{V}^{b(\beta+1) p}(x) \Delta_{V}^{\alpha}(x) d x \\
\quad=\int_{\left\langle\alpha_{n} z_{0}, n z_{0}\right\rangle} \Delta_{V}^{-1}(x) d x .
\end{aligned}
$$


Since $\beta+1<0$, it follows that $\alpha_{n} \rightarrow 0$ and $\left\langle\alpha_{n} z_{0}, n z_{0}\right\rangle \nearrow V$ as $n \rightarrow \infty$. Noting that $\Delta_{V}^{-1}(x)$ is not integrable on $V$, we have that

$$
\int_{V} f_{n}^{p}(x) \Delta_{V}^{\alpha}(x) d x \rightarrow \infty \quad \text { as } n \rightarrow \infty .
$$

Using Fubini's theorem and noting that $\int_{\langle x, \infty\rangle} \Delta_{V}^{\beta}(y) d y$ is finite for every $x \in V$, we have

$$
\begin{aligned}
& \int_{V}\left(\int_{\langle 0, y\rangle} f_{n}(z) \Delta_{V}^{\alpha}(z) d z\right)^{q} \Delta_{V}^{\beta}(y) d y \\
& \quad=\int_{V}\left(\int_{\langle 0, y\rangle} f_{n}(x) \Delta_{V}^{\alpha}(x)\left(\int_{\langle 0, y\rangle} f_{n}(z) \Delta_{V}^{\alpha}(z) d z\right)^{q-1} d x\right) \Delta_{V}^{\beta}(y) d y \\
& \quad \geq \int_{V}\left(\int_{\langle 0, y\rangle} f_{n}(x) \Delta_{V}^{\alpha}(x)\left(\int_{\langle 0, x\rangle} f_{n}(z) \Delta_{V}^{\alpha}(z) d z\right)^{q-1} d x\right) \Delta_{V}^{\beta}(y) d y \\
& \quad=\int_{V} f_{n}(x) \Delta_{V}^{\alpha}(x)\left(\int_{\langle 0, x\rangle} f_{n}(z) \Delta_{V}^{\alpha}(z) d z\right)^{q-1}\left(\int_{\langle x, \infty\rangle} \Delta_{V}^{\beta}(y) d y\right) d x \\
& \quad=c \int_{V} f_{n}(x) \Delta_{V}^{\alpha}(x)\left(\int_{\langle 0, x\rangle} f_{n}(z) \Delta_{V}^{\alpha}(z) d z\right)^{q-1} \Delta_{V}^{\beta+1}(x) d x,
\end{aligned}
$$

where $c$ is a constant independent of $f_{n}$.

Since $\beta+1<0, f_{n}(x) \Delta_{V}^{-a(\alpha+1)}(x)$ is a decreasing function of $x \in V$ in the partial ordering defined by $V$. Further, we have that

$$
\begin{aligned}
& \int_{\langle 0, x\rangle} f_{n}(z) \Delta_{V}^{\alpha}(z) d z \\
& \quad= \int_{\langle 0, x\rangle} f_{n}(z) \Delta_{V}^{\alpha}(z) \Delta_{V}^{-a(\alpha+1)}(z) \Delta_{V}^{a(\alpha+1)}(z) d z \\
& \quad \geq f_{n}(x) \Delta_{V}^{-a(\alpha+1)}(x) \int_{\langle 0, x\rangle} \Delta_{V}^{a(\alpha+1)+\alpha}(z) d z
\end{aligned}
$$

By (7), in order that $\int_{\langle 0, x\rangle} \Delta_{V}^{\alpha}(z) d z$ be finite, it is necessary that $\alpha>-1$. Thus, $a(\alpha+1)>0$ and $\int_{\langle 0, x\rangle} \Delta_{V}^{a(\alpha+1)+\alpha}(z) d z$ is finite. So the last integral in (10) equals $c \Delta_{V}^{(\alpha+1)(a+1)}(x)$ and

$$
\int_{\langle 0, x\rangle} f_{n}(z) \Delta_{V}^{\alpha}(z) d z \geq c f_{n}(x) \Delta_{V}^{\alpha+1}(x)
$$

where $c$ does not depend on $f_{n}$. 
Therefore, (9) becomes

$$
\begin{aligned}
& \int_{V}\left(\int_{\langle 0, y\rangle} f_{n}(z) \Delta_{V}^{\alpha}(z) d z\right)^{q} \Delta_{V}^{\beta}(y) d y \\
& \quad \geq c \int_{V} f_{n}(x) \Delta_{V}^{\alpha}(x)\left(\int_{\langle 0, x\rangle} f_{n}(z) \Delta_{V}^{\alpha}(z) d z\right)^{q-1} \Delta_{V}^{\beta+1}(x) d x \\
& \quad \geq c \int_{V} f_{n}(x) \Delta_{V}^{\alpha}(x) f_{n}^{q-1}(x) \Delta_{V}^{(\alpha+1)(q-1)}(x) \Delta_{V}^{\beta+1}(x) d x .
\end{aligned}
$$

Noting that

$$
\Delta_{V}^{(\alpha+1)(q-1)+(\beta+1)}(x) \geq f_{n}^{p-q}(x)
$$

we finally have

$$
\int_{V}\left(\int_{\langle 0, y\rangle} f_{n}(z) \Delta_{V}^{\alpha}(z) d z\right)^{q} \Delta_{V}^{\beta}(y) d y \geq c \int_{V} f_{n}^{p}(x) \Delta_{V}^{\alpha}(x) d x .
$$

Therefore,

$$
\left(\int_{V}\left(\int_{\langle 0, y\rangle} f_{n}(z) \Delta_{V}^{\alpha}(z) d z\right)^{q} \Delta_{V}^{\beta}(y) d y\right)^{1 / q} \geq c\left(\int_{V} f_{n}^{p}(x) \Delta_{V}^{\alpha}(x) d x\right)^{1 / q},
$$

where $c$ is independent of $f_{n}(x)$. Since $\int_{V} f_{n}^{p}(x) \Delta_{V}^{\alpha}(x) d x \rightarrow \infty$ as $n \rightarrow \infty$ and $q<p$, there is no constant $c$ such that for all $f_{n}$,

$$
\left(\int_{V}\left(\int_{\langle 0, y\rangle} f_{n}(z) \Delta_{V}^{\alpha}(z) d z\right)^{q} \Delta_{V}^{\beta}(y) d y\right)^{1 / q} \leq c\left(\int_{V} f_{n}^{p}(x) \Delta_{V}^{\alpha}(x) d x\right)^{1 / p} .
$$

So we proved Theorem 4 in the case $1<q$. If $q=1$, we define

$$
f_{n}(x)=\min \left(n, \Delta_{V}^{b(\beta+1)}(x)\right) \chi_{\left\langle 0, n z_{0}\right\rangle}(x), \quad x \in V, n=1,2, \ldots,
$$

and (9) becomes the following simple inequality:

$$
\begin{aligned}
& \int_{V}\left(\int_{\langle 0, y\rangle} f_{n}(z) \Delta_{V}^{\alpha}(z) d z\right) \Delta_{V}^{\beta}(y) d y \\
& \quad=\int_{V} f_{n}(x) \Delta_{V}^{\alpha}(x)\left(\int_{\langle x, \infty\rangle} \Delta_{V}^{\beta}(y) d y\right) d x \\
& \quad=c \int_{V} f_{n}(x) \Delta_{V}^{\alpha+\beta+1}(x) d x \geq c \int_{V} f_{n}^{p}(x) \Delta_{V}^{\alpha}(x) d x .
\end{aligned}
$$

The theorem is proved.

Now we consider the Hardy operator of the form

$$
\tilde{H}_{\alpha} f(x)=\int_{\langle x, \infty\rangle} f(t) \Delta_{V}^{\alpha}(t) d t .
$$

For $\widetilde{H}_{\alpha}$ we expect the following similar result: 
Theorem 5. Let $V$ be a domain of positivity in $R^{d}$. If $1 \leq q<p<\infty$, then for any values of $\alpha$ and $\beta$, there is no constant $c>0$ such that

$$
\left(\int_{V} \Delta_{V}^{\beta}(x)\left(\tilde{H}_{\alpha} f(x)\right)^{q} d x\right)^{1 / q} \leq c\left(\int_{V} f^{p}(x) \Delta_{V}^{\alpha}(x) d x\right)^{1 / p}
$$

holds for all $f \geq 0$.

Proof. Assume that for some $\alpha$ and $\beta$ there is a constant $c>0$ such that (11) holds for all $f \geq 0$. Let $g \geq 0$ be a function defined on $V$ with $\int_{V} g^{p}(y) \Delta_{V}^{\alpha}(y) d y=1$. Then, for $f \geq 0$,

$$
\begin{aligned}
& \int_{V}\left(\int_{\langle 0, y\rangle} f(x) \Delta_{V}^{\beta}(x) d x\right) g(y) \Delta_{V}^{\alpha}(y) d y \\
& \quad=\int_{V}\left(\int_{\langle x, \infty\rangle} g(y) \Delta_{V}^{\alpha}(y) d y\right) f(x) \Delta_{V}^{\beta}(x) d x \\
& \quad \leq\left(\int_{V}\left(\int_{\langle x, \infty\rangle} g(y) \Delta_{V}^{\alpha}(y) d y\right)^{q} \Delta_{V}^{\beta}(x) d x\right)^{1 / q}\left(\int_{V} f^{q^{\prime}}(y) \Delta_{V}^{\beta}(y) d y\right)^{1 / q^{\prime}} \\
& \quad \leq c\left(\int_{V} g^{p}(y) \Delta_{V}^{\alpha}(y) d y\right)^{1 / p}\left(\int_{V} f^{q^{\prime}}(y) \Delta_{V}^{\beta}(y) d y\right)^{1 / q^{\prime}} \\
& \quad=c\left(\int_{V} f^{q^{\prime}}(y) \Delta_{V}^{\beta}(y) d y\right)^{1 / q^{\prime}} .
\end{aligned}
$$

Thus, for $f \geq 0$,

$$
\left(\int_{V} \Delta_{V}^{\alpha}(y)\left(\int_{\langle 0, y\rangle} f(x) \Delta_{V}^{\beta}(x) d x\right)^{p^{\prime}} d y\right)^{1 / p^{\prime}} \leq c\left(\int_{V} f^{q^{\prime}}(y) \Delta_{V}^{\beta}(y) d y\right)^{1 / q^{\prime}} .
$$

But this is impossible by Theorem 4. So there are no $\alpha$ and $\beta$ so that (11) holds for all $f \geq 0$.

\section{REFERENCES}

1. S. G. Gindikin, Analysis in homogeneous domains, Russian Math. Surveys 19 (1964), 1-89.

2. M. Koecher, Positivitätsbereiche im $R^{n}$, Amer. J. Math. 79 (1957), 575-596. (German)

3. V. G. Mazja, Sobolev spaces, Springer-Verlag, 1985, pp. 39-51.

4. T. Ostrogorski, Analogues of Hardy's inequality in $R^{n}$, Studia Math. 88 (1988), 209-219.

5. R. T. Rockafellar, Convex analysis, Princeton Univ. Press, 1970, pp. 95-101.

6. O. Rothaus, Domains of positivity, Abh. Math. Sem. Univ. Hamburg 24 (1960), 189-235.

7. Y. Sagher, M. V. Siadat, and K. Zhou, Norm inequalities for integral operators on cones, Colloq. Math. 60/61 (1990).

8. E. Sawyer, Weighted Lebesgue and Lorentz norm inequalities for the Hardy operator, Trans. Amer. Math. Soc. 281 (1984).

Department of Mathematics, California State University, Sacramento, California 95819-6051 